\title{
Fluidic Vectoring of a Planar Incompressible Jet Flow
}

\author{
Miguel Alfonso Mendez ${ }^{1, \star}$, Maria Teresa Scelzo ${ }^{1}$, Adriana Enache ${ }^{1}$, and Jean-Marie Buchlin ${ }^{1}$ \\ ${ }^{1}$ EA Dep., von Karman Institute for Fluid Dynamics, Sint-Genesius-Rode, Belgium
}

\begin{abstract}
This paper presents an experimental, a numerical and a theoretical analysis of the performances of a fluidic vectoring device for controlling the direction of a turbulent, bi-dimensional and low Mach number (incompressible) jet flow. The investigated design is the co-flow secondary injection with Coanda surface, which allows for vectoring angles up to $25^{\circ}$ with no need of moving mechanical parts. A simple empirical model of the vectoring process is presented and validated via experimental and numerical data. The experiments consist of flow visualization and image processing for the automatic detection of the jet centerline; the numerical simulations are carried out solving the Unsteady Reynolds Average Navier- Stokes (URANS) closed with the $k-\omega$ SST turbulence model, using the PisoFoam solver from OpenFOAM. The experimental validation on three different geometrical configurations has shown that the model is capable of providing a fast and reliable evaluation of the device performance as a function of the operating conditions.
\end{abstract}

\section{Introduction}

Controlling the direction of a jet flow is a crucial operation in several engineering applications. Examples range from thrust control in propulsion systems [1-4] to particle sorting in powder-based industrial processes [5, 6].

Such control is known as jet vectoring and can be mechanic, that is by means of movable surfaces, or aerodynamic, that is by means of a secondary injection or suction. While the mechanic vectoring is always effective and offers large vectoring performances, the presence of moving mechanical parts leads to control devices that are complex, heavy, aerodynamically inefficient and hard to integrate on existing nozzles [7]. By contrast, aerodynamic systems are light and simple to integrate, but their vectoring performance and controllability are limited to a relatively narrower range of operating conditions, which depends on the particular scheme adopted [8-10].

These systems, referred to as fluidic vectoring devices, exploit the interaction of the jet flow with adjacent secondary fluid streams and can be classified depending on how such interaction is generated.

Fluidic vectoring devices can operate in steady or unsteady conditions. Devices that operate in steady conditions can be further classified based on the direction of the secondary flow, which can be in co-flow (blowing, $[4,11]$ ), counter-flow (suctioning, [12-14]) or a suitable combination of both (blowing and suctioning, [6, 15]). These designs usually combine the secondary flow with a smooth extension of the nozzle to exploit the well known Coanda effect, that is the adherence of a fluid stream to a wall, as a result of an unbalance in the entrainment of surrounding fluid [16-18]. Devices that operate in unsteady conditions

\footnotetext{
^e-mail: mendez@vki.ac.be
}

can be further classified based on the net mass flux introduced, which can be zero if the secondary stream is produced by synthetic jets $[9,19]$ or not if it is produced by pulsing jets $[20,21]$.

Regardless of the fluidic scheme adopted, the design and the control of any aerodynamic vectoring system require a precise evaluation of its vectoring performance as a function of the operating conditions. The scope of this work is to present a complete characterization of an aerodynamic vectoring system combining experimental, numerical and theoretical analysis.

In particular, the investigated design is the Coanda coflow fluidic scheme, to be implemented in the vectoring of a turbulent bi-dimensional jet flow at low speed, namely at a Mach number sufficiently low to neglect compressibility effects. This design allows for avoiding the use of highfrequency actuators as in synthetic jets vectoring, or the installation of injection and suction lines at a short distance from the jet. On the other hand, its use for low Mach number flows is not documented in the literature. Although several experimental works have been presented [4, 22], a definition of the role of its geometrical and operational parameters is still lacking and most of the numerical and analytical modeling attempts are focused on the high-speed regimes typical of aircraft propulsion [23-28].

To support design engineers in the development of fluidic vectoring schemes, this work proposes an empirical model of the jet deflection, developed on the basis of simple physical considerations and validated via numerical and experimental data. Such model does not aim to exhaustively describe all the physical mechanisms involved in the vectoring process but to provide an engineering tool to analyze the impact of the operating parameters on the 
system performance and to provide a physical insight on the vectoring phenomenon.

The investigated co-flow fluidic design is described in Section 2, along with the proposed empirical model. A set of 2D simulations were used to support the simplifying assumptions in the model derivation, using Unsteady Reynolds Average Navier Stokes (RANS) modeling. The numerical set up is presented in Section 3. Finally, the empirical model is validated experimentally, by combining flow visualization and image processing, as described in Section 4. The results are presented and discussed in Section 5; the conclusions are drawn in Section 6.

\section{Problem Formulation}

The co-flow fluidic vectoring design under consideration is shown in the schematic in Fig.1, which recalls the main control variable: the opening for the primary and the secondary jets $h_{p}$ and $h_{s}$, their respective average velocities $u_{p}$ and $u_{s}$ and the radius of the Coanda surface $R$, which is tangent to the secondary channel. The distance $L$ between the primary and the secondary channels should be minimized, compatibly with mechanical constraints, to promote the interaction between the two jets.

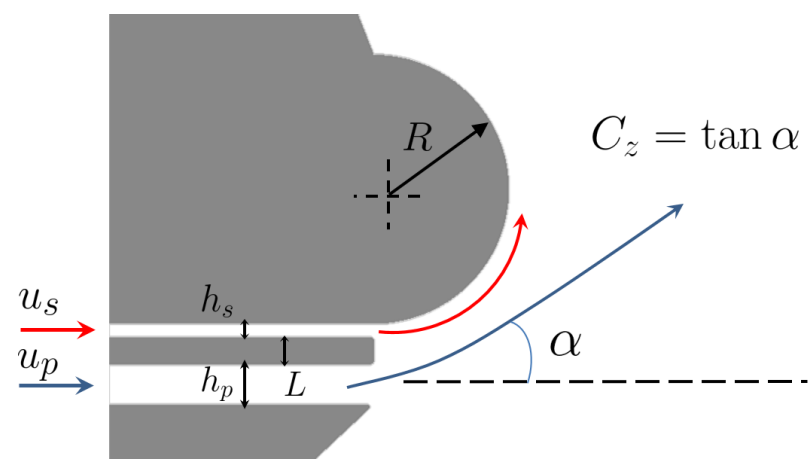

Fig. 1. Geometrical configuration and operating parameters for the co-flow fluidic vectoring considered in this work.

For a given fluidic nozzle, that is fixing $h_{s}, h_{p}, L, R$, the operating condition of the device is typically given in terms of mass flux ratio $\beta$ or momentum ratio $\beta_{M}$. For an incompressible flow configuration, with the density constant and equal in both the injections, these are

$$
\beta=\frac{h_{s} u_{s}}{h_{p} u_{p}} ; \quad \beta_{M}=\frac{h_{s} u_{s}^{2}}{h_{p} u_{p}^{2}}=\beta \frac{u_{s}}{u_{p}}=\beta^{2} \frac{h_{p}}{h_{s}} .
$$

Although these quantities are interchangeable, the momentum ratio $\beta_{M}$ is considered to better represent the physics of the vectoring process and it is hereinafter chosen as the dimensionless parameter describing the operation condition.

The performance of the device, i.e its operational map, is typically defined in terms of thrust vectoring coefficient, that is the slope $C_{Z}=\tan (\alpha)$ of the achieved vectoring angle $\alpha$, as function of the momentum ratio $\beta_{M}$. This operational map is qualitatively divided in three zones [4, 28], referred to as dead zone, control zone and saturation zone.

The dead zone is the region where the momentum ratio $\beta_{M}$ is below a critical value $\beta_{M}^{C}$, and is characterized by no jet vectoring or even negative vectoring ( $\alpha<0$ in Fig.1). The saturation zone is the region above a critical value $\beta_{M}^{S}$, where the vectoring of the jet no longer increases with increased $\beta_{M}$. The co-flow device, therefore, can operate within a range $\beta_{M} \in\left[\beta_{M}^{C}, \beta_{M}^{S}\right]$ which depends on its geometry, in the so called control zone.

The empirical model proposed in this work hinges upon the formulation of three physical principles, each described in a dedicated subsection.

First, a general vectoring law is formulated in Section 2.1 by modeling the jet deflection as the result of a transversal pressure gradient on the primary jet flow. This pressure gradient originates from the Coanda effect acting on the secondary jet flow, as formulated in Section 2.2. Depending on both the nozzle geometry and the operating condition, the interaction between the two jets strongly influences the vectoring physics, as formulated in Section 2.3. Section 2.4 finally collects all the theoretical considerations into a simple equation to describe the operational map of the device.

The flow of both jets is assumed to be incompressible, isothermal and mono-dimensional, that is concentrated along their respective centerlines. Turbulent and viscous effects are introduced empirically.

\subsection{Flow deflection of the Primary Jet}

In a Lagrangian framework, neglecting the effects of viscosity, the forces acting on a control fluid volume $\Omega$ of density $\rho$, traveling at the flow velocity $u_{M}(\zeta)$, only includes inertia and pressure gradient as shown in the schematic of Fig.2.

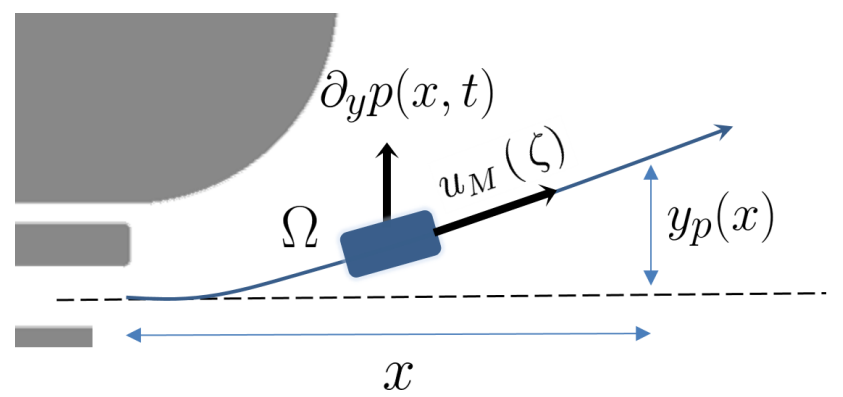

Fig. 2. Force balance of a control volume in the primary jet, advected at the averaged velocity $u_{M}$ and traveling under the action of a transversal pressure gradient $\partial_{y} p$. The trajectory of jet becomes linear after a short distance.

Here the flow velocity $u_{M}(\zeta)$ is an average of the velocity of the two injections, described in Section 2.3, and $\zeta$ is the curvilinear coordinate following the primary jet centerline. In a first approximation, we neglect the curvature effect on the primary jet, which is on the other hand crucial 
for the secondary jet as described in Section2.2. Assuming that the jet travels along a line inclined by the vectoring angle $\alpha$, the link between the curvilinear coordinate and the horizontal axis is simply $x \approx \zeta / \cos (\alpha)$.

As the flow is released in atmosphere (i.e. is sufficiently far from the walls) the stream-wise pressure gradient is negligible and the force balance only includes a transversal pressure gradient $\partial_{y} p(x)$ :

$$
\rho \Omega \frac{D^{2} y_{p}(x)}{D t^{2}}=\Omega \partial_{y} p(x, t),
$$

where $y_{p}(x)$ is the vertical displacement of the control volume with respect to the origin, located at the nozzle geometrical centerline. Introducing the material derivative $D(\bullet) / D t=\partial_{t}(\bullet)+u_{p}(x) \partial_{x}(\bullet)$, eq. (2) becomes:

$$
\partial_{t t} y_{p}+2 u_{M} \partial_{x t} y_{p}+u_{M}^{2} \partial_{x x} y_{p}=-\frac{1}{\rho} \partial_{y} p(x, t) .
$$

The complete solution of eq.(3) by means of Laplace transform is proposed in [29]. In this work, considering only the steady solution reached at the end of the transitory, the time derivatives can be dropped and eq.(3) is easily integrated to retrieve the trajectory of the jet centerline:

$$
\begin{aligned}
\partial_{x x} y_{p}(x) & =\frac{1}{\rho} \frac{\partial_{y} p(x)}{u_{M}^{2}(x)} \\
\partial_{x} y_{p}(x) & =C_{Z}=\int_{0}^{x} \frac{1}{\rho} \frac{\partial_{y} p(s)}{u_{M}^{2}(s)} d s+C_{1} \\
y_{p}(x) & =\int_{0}^{x} \int_{0}^{x} \frac{1}{\rho} \frac{\partial_{y} p(s)}{u_{M}^{2}(s)} d s+C_{1} x+C_{2}
\end{aligned}
$$

The integration constants $C_{1}$ and $C_{2}$ can be set to zero by assuming that the primary jet flow is infinitely 'rigid' at the nozzle outlet, and thus $\partial_{x} y_{p}=y_{p}=0$ at $x=0$.

Equations (4) allows for several important considerations on the thrust vectoring phenomenon. First, no thrust vectoring is produced if the pressure gradient $\partial_{y} p$ is identically null over the entire domain. Second, provided that the pressure gradient only acts within a certain region, say $\partial_{y} p(x)=0 \forall x>x^{*}$, eq.(4c) leads to a constant value, that is the trajectory of the jet flow $y_{p}(x)$ becomes a straight line with constant slope:

$$
C_{Z}\left(\beta_{M}\right)=\int_{0}^{x^{*}} \frac{1}{\rho} \frac{\partial_{y} p\left(s, \beta_{M}\right)}{u_{M}^{2}\left(s, \beta_{M}\right)} d s .
$$

This equation can be considered as a general vectoring law, which shows that the vectoring performance depends on the strength $\partial_{y} p\left(x, \beta_{M}\right)$ and the extension $x^{*}$ of the pressure gradient, and on the averaged advection velocity of the jet $u_{M}\left(x, \beta_{M}\right)$. In principle, then, a faster jet requires a stronger (or more extended) pressure gradient to achieve the same vectoring angle. Moreover, the characteristic curve $C_{Z}\left(\beta_{M}\right)$ of the device can be inferred from the trends of these two terms as function of the momentum ratio $\beta_{M}$ : the control zone can be seen as the region in which $\partial_{y} p\left(\beta_{M}\right)$ grows faster than $u_{M}^{2}\left(\beta_{M}\right)$, while saturation occurs then these two quantities have the same functional dependency on $\beta_{M}$, so that their ratio remains constant. These two terms are analyzed in Section 2.2 and 2.3 respectively.

\subsection{Vectoring performance of the Secondary Jet}

As long as the secondary jet flow is attached on the cylindrical surface, a pressure drop is produced at the wall. This phenomenon, known as the Coanda effect, is usually described from the force balance of an inviscid fluid [16-18], which is shown in the schematic of Figure 3.

As the velocity is uniform along the radial direction, the force balance of a fluid element at a distance $r$ from the Coanda center reads:

$$
\partial_{r} p=\frac{\rho u_{s}^{2}}{r}
$$

Integrating eq.(6) over the thickness of the secondary jet, assumed to remain $\sim h_{s}$, yields the pressure drop over its cross section:

$$
\Delta P_{c}=\rho u_{s}^{2}(\theta) \int_{R}^{R+h_{s}} \frac{d r}{r}=\rho u_{s}^{2} \ln \left(\frac{R+h_{s}}{R}\right)
$$

This result was used in a first attempt to define the pressure gradient driving the vectoring as the average value $\partial_{y} p \sim \Delta P_{c} / h_{s}$. In the range of geometrical parameters, however, a better reference value was found to be the maximum pressure gradient produced at the wall, i.e. taking $r=R$ in eq.(6):

$$
\left.\partial_{r} p\right|_{r=R}=\frac{\rho u_{s}^{2}}{R} .
$$

Any result provided by inviscid theory provides nevertheless an upper and ideal limit for the achievable pressure gradient. Viscosity and turbulence diffusion, in fact, introduce a non-uniform velocity profile, a decay of its maximum value along the polar coordinate and a thickening of the jet boundary $h_{s}$. An analytical, self-similar solution

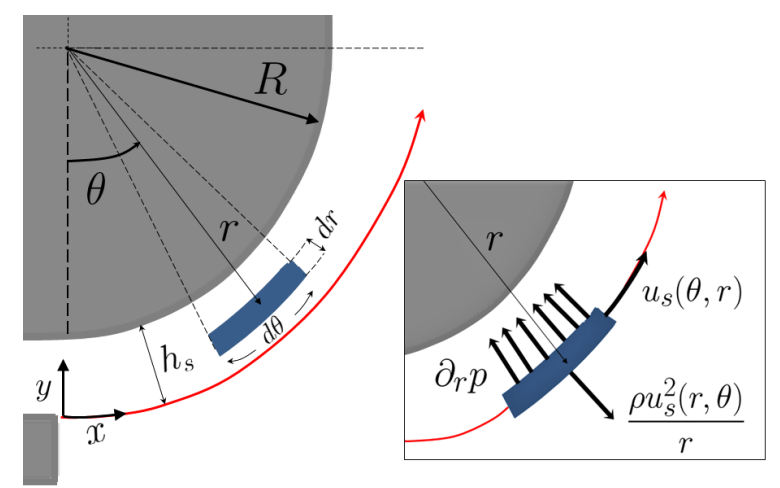

Fig. 3. Radial equilibrium of a control volume in the secondary jet flow, attached on the cylindrical surface by the Coanda effect. The flow is assumed to be inviscid, so that the velocity profile is constant and the jet thickness remains $\sim h_{s}$. 
which describes the entire evolution of a Coanda wall jet is proposed in [30]. For the purpose of this work, we postulate that eq.(8) requires at least two empirical corrections, written in variable separated form as

$$
\partial_{y} p \approx\left(\frac{\rho u_{s}^{2}}{R}\right) c_{1}\left(\frac{h_{s}}{R}, h_{p}, R e_{s}, \beta_{M}\right) P(x) .
$$

The first correction $c_{1}$ takes into account the reduction due to the curvature ratio $h_{s} / R$, a term often referred to as curvature parameter [30], turbulence and viscous diffusion, and the momentum exchange with the primary jet flow. This term depends on the geometrical parameters $h_{s} / R, h_{p}$, on the Reynolds number of the secondary injection $\operatorname{Re}_{s}=h_{s} u_{s} / v$, with $v$ the kinematic viscosity, and the blowing ratio $\beta_{M}$. In a first simplification, however, we assume that this correction is solely a function of the geometrical parameters.

The second correction $P(x)$ takes into account the decay law of the net pressure gradient, due to both the dissipation and the momentum exchange between the two jets. This function should vary in the range $P \in[0,1]$ and lead to a vanishing of the suctioning effect above a certain value $x^{*}$ from the inlet. An attempt to define it from URANS simulations is presented in Section 5.1. Here, we limit the analysis of the pressure gradient to the introduction of the momentum ratio in eq.(9):

$$
\frac{\partial_{y} p(x, \beta)}{\rho} \approx \beta_{M} u_{p}^{2} \frac{h_{p}}{h_{s}} c_{1}\left(\frac{h_{s}}{R}, h_{p}\right) \frac{1}{R} P(x) .
$$

That is, if the dependency of the correction $c_{1}$ on the blowing ratio is neglected, the pressure gradient grows linearly with $\beta_{M}$ for a given velocity in the primary jet flow.

\subsection{Momentum exchange between Jet Flows}

The average velocity in eq.(5) takes into account the momentum exchange between the two jets. An approach solely based on the momentum conservation appeared nevertheless particularly cumbersome since it requires the evaluation of the viscous dissipation on the Coanda surface and a clear definition of the jet flow boundaries. This approach is therefore dropped in favor of a more heuristic average, which takes the form

$$
u_{M}^{2}=\xi\left(\beta_{M}\right) u_{s}^{2}+\left[1-\xi\left(\beta_{M}\right)\right] u_{p}^{2}
$$

The mixing coefficient $\xi\left(\beta_{M}\right)$ takes into account that at low momentum ratios $\left(\beta_{M} \ll 1\right)$, when $u_{s} \ll u_{p}$, the average velocity must be close to that of the primary (i.e. $\xi \approx 0)$ while at large momentum ratios $\left(\beta_{M}>1\right)$, when $u_{s} \gg u_{p}$, the average velocity must be close to that of the secondary (i.e. $\xi \approx 1$ ).

Observing that the pressure gradient driving the jet vectoring in eq.(9) is approximately $\partial_{y} p \propto u_{s}^{2}$, one could see that the condition $\xi=1$ defines the saturation of the device, since it yields $u_{M}^{2}=u_{s}^{2}$ and the velocity of the secondary jet -that is the operating conditions- cancels out from eq.(5). Introducing the momentum ratio in eq.(12), and introducing a shape function $G(x)$ for the stream-wise velocity evolution yields:

$$
u_{M}^{2}(x, \beta)=u_{p}^{2}\left[\xi\left(\beta_{M}\right) \frac{h_{p}}{h_{s}} \beta_{M}+1-\xi\left(\beta_{M}\right)\right] G^{2}(x) .
$$

The shape function $G(x)$ ranges from 1 , (for $x \rightarrow 0$ ) to 0 (for $x \rightarrow \infty$ ). Eq.(12) can be seen as a variable separated form of the average velocity, of the kind often used in the study of self-similar flows.

For a turbulent free jet released at a velocity $u_{j}$, for example, this shape function $G_{j}(x)=u_{j}(x) / u_{j}$ is available in the framework of boundary layer theory, using a mixing length turbulence model [29, 31-33]:

$$
G_{j}(x)=\left\{\begin{array}{l}
G_{j}=1 \quad \text { if } x \leq x_{c} \\
G_{j}(x)=\left(x_{c} / x\right)^{0.5} \quad \text { if } x>x_{c}
\end{array}\right.
$$

where $x_{c}$ is the length of the potential core, typically of the order of $2-3$ times the nozzle opening.

A similar approach could in principle be followed for both the primary and the secondary jet flows, and use the averaging process point-wise. Here, however, we make no analytical attempt in defining a general function $G(x)$, nor in seeking for a self-similar form of both jets during their interaction. This shape function is presented for several numerical test cases in Section 5.1. It is nevertheless worth observing that the influence of the average decay $G(x)$ becomes important in eq.(5) only as long as the length of the 'potential core' of the two flows is much shorter than the critical position $x^{*}$ within which the pressure gradient acts, appearing in the upper limit of the integral.

\subsection{Model Formulation}

Introducing eq. (12) and (10) into in eq.(5) leads to the final form of the empirical model proposed for the thrust vectoring effect:

$$
C_{Z}\left(\beta_{M}\right)=C\left(h_{s}, h_{p}, R\right) \frac{\beta_{M}}{\xi\left(\beta_{M}\right) \frac{h_{p}}{h_{s}} \beta_{M}+1-\xi\left(\beta_{M}\right)},
$$

with the model coefficient

$$
C=\frac{h_{s}}{h_{p}} c_{1}\left(h_{s}, h_{p}, R\right) \frac{1}{R} \int_{0}^{x^{*}} \frac{F(s)}{G^{2}(s)} d s
$$

This coefficient is assumed to be independent from the operating condition and thus defined by a given nozzle design. An evaluation of such assumption is provided in Section 5.1. Here, the model closure requires the definition of the functional $\xi\left(\beta_{M}\right)$ and the constraints required for its identification. The procedure proposed is based on the observation that eq.(16), or more generally (5), allows for a saturation of the vectoring angle provided that the average velocity becomes $u_{M} \sim u_{s}$, that is $\xi \approx 1$. To close the model, therefore, we write the functional $\xi\left(\beta_{M}\right)$ as a polynomial expansion in $\beta_{M}$ of order $O(P)$ : 


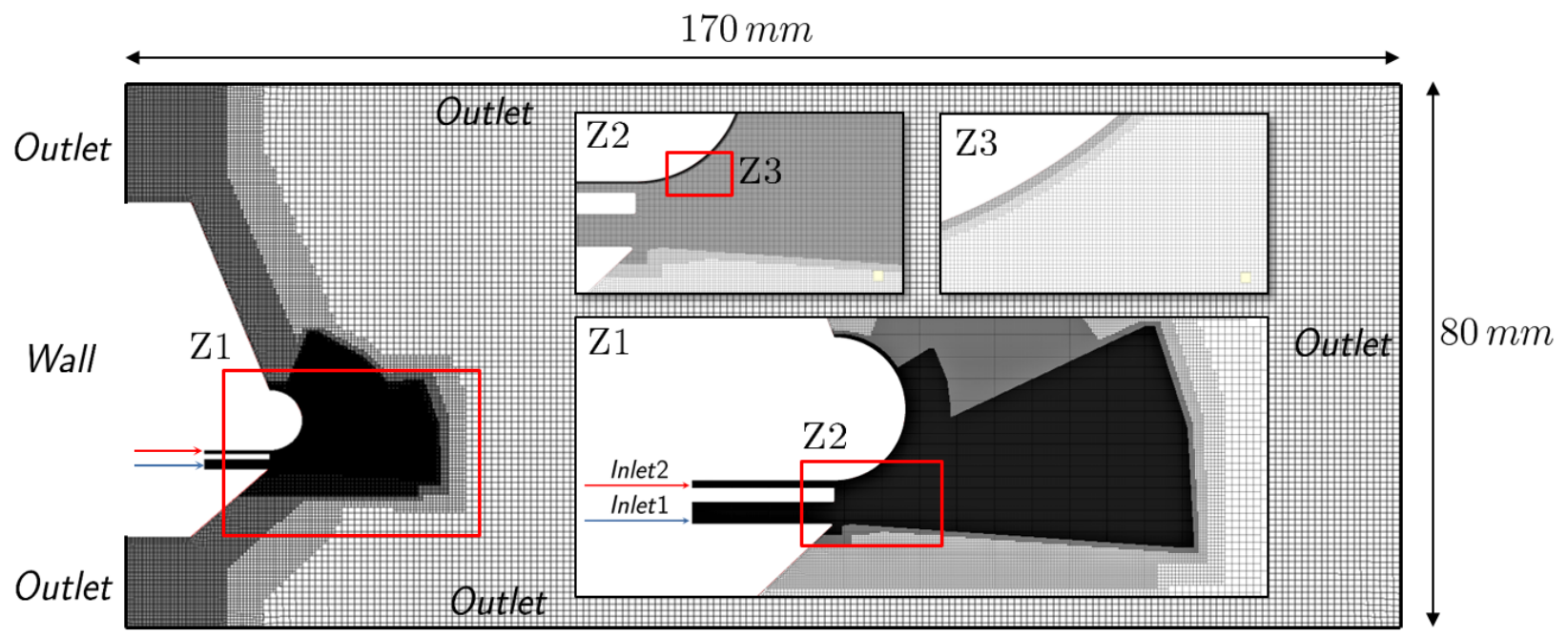

Fig. 4. Computational domain and discretization mesh, with the boundary conditions in each patch. The different level of mesh refinements are shown in three zooms $(Z 1, Z 2$ and $Z 3)$. The mesh consists of about $2 \times 10^{5}$ cells, and the domain is sufficiently large to simulate the entire evolution of the jets before they reach the numerical boundaries.

$$
\xi\left(\beta_{M}\right)=\sum_{j=1}^{j=P} a_{j} \beta_{M}^{j},
$$

defined so that $\xi\left(\beta_{M}=0\right)=0$, and constrained so that $\xi\left(\beta_{S}\right)=1$. As the order of the expansion is increased above one, a set of $n-1$ constraints on its derivatives must be defined. A plausible hypothesis is that the growth of $\xi$ saturates as it approaches critical value $\beta_{M}^{S}$ for which $\partial_{\beta_{M}^{s}} C_{Z}=0$, and so $\partial_{\beta_{M}}^{n} \xi\left(\beta_{M}^{S}\right)=0$ for any derivative $n>1$. The opposite approach is to postulate an initially gentle growth of $\xi\left(\beta_{M}\right)$ followed by a strong growth towards $\beta_{M}^{S}$, that is $\partial_{\beta_{M}}^{n} \xi\left(\beta_{M}^{C}\right)=0$ for $n>1$.

In what follows, we illustrate the procedure only for the first order expansion, which provided a sufficiently accurate comparison with the presented experimental data. Assuming that $\xi=a_{1} \beta_{M}$, eq.(16) becomes:

$$
C_{Z}\left(\beta_{M}\right)=C\left(h_{s}, h_{p}, R\right) \frac{\beta_{M}}{a_{1} \frac{h_{p}}{h_{s}} \beta_{M}^{2}-a_{1} \beta_{M}+1}
$$

The maximum deflection $\left(\partial_{\beta_{M}} C_{Z}=0\right)$ is therefore obtained when

$$
\beta_{M}^{S}=\sqrt{\frac{h_{s}}{h_{p} a_{1}}}
$$

Imposing that this corresponds to $\xi=1$, the coefficient becomes $a_{1}=h_{p} / h_{s}$. The model in eq.(16) reduces therefore only to one unknown, assumed to be independent from the operating conditions. Interestingly, the momentum ratio at which the maximum deflection occurs becomes $\beta_{S}=h_{s} / h_{p}$, and the corresponding maximum vectoring coefficient is $C_{Z}\left(\beta_{S}\right)=C h_{s} / h_{p}$. The model coefficient $C$ is deduced by fitting eq.(16), with $a_{1}=h_{p} / h_{s}$, to the experimental points in Section 5.2; the terms in eq.(14) and their dependency on the operating conditions is investigated in Section 5.1. Remarkably, such simple model predicts a negligible impact of the Coanda radius, and a much stronger influence of the opening ratio $h_{p} / h_{s}$.

\section{Numerical Methods}

The 2D numeral simulations supporting the development of the proposed engineering model are performed using a classical Unsteady Reynolds Averaged Navier Stokes (URANS) approach, implemented in the PisoFOAM solver in OpenFOAM with the well-established pressure-based finite-volume methodology.

The computational domain and the mesh discretization are shown in Figure 4, together with the definition of the boundary conditions. The domain is created using the open-source Free $C A D$ software, and consists of a rectangular box of $170 \times 80 \mathrm{~mm}^{2}$ from which the nozzle geometry is removed via Boolean subtraction. The size of the domain is large enough to allow for the complete development of the two jets and their interaction, as well as for simulating the entrainment flow from the external boundaries without undesired boundary effects.

The discretization mesh is unstructured, prepared using the HEXPRESS $S^{T M}$ package from Numeca and consisting of about with an $2 \times 10^{5}$ polyhedral cells, with different levels of refinement in the entrainment regions, the zone of jet mixing and development (cf. Fig.4, zoomed view Z1), and the Coanda wall (cf. Fig.4, zoomed views Z2 and Z3). Here, in particular, the mesh allows for an average $y^{+} \approx 1$ and no wall function are employed. The URANS equations are therefore solved up to the wall, using the well-known $k-\omega S S T$ turbulence model [34].

All the wall patches are set to no-slip condition, with a zero gradient condition for the pressure, a large value of specific dissipation $\omega$, and a zero turbulence kinetic energy $k$. In the outlet patches, a zero gradient condition is imposed to both the turbulence quantities and the 


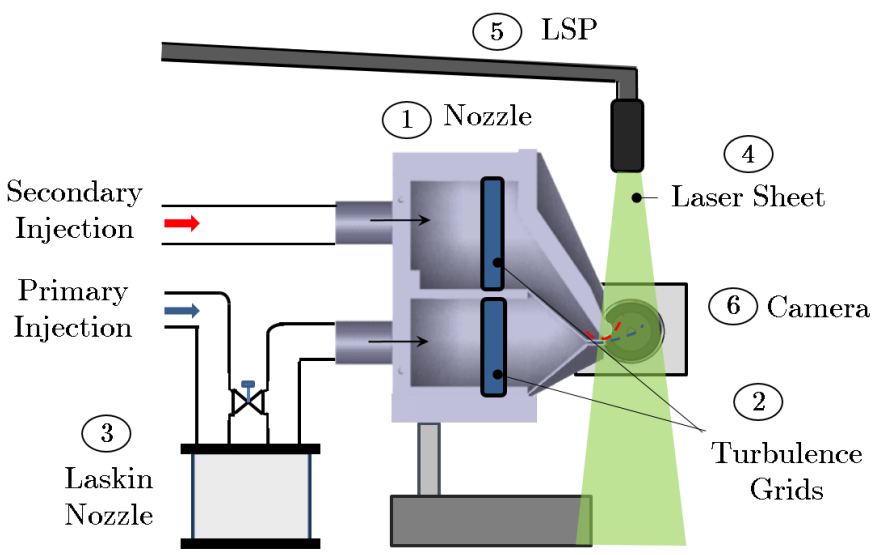

a)

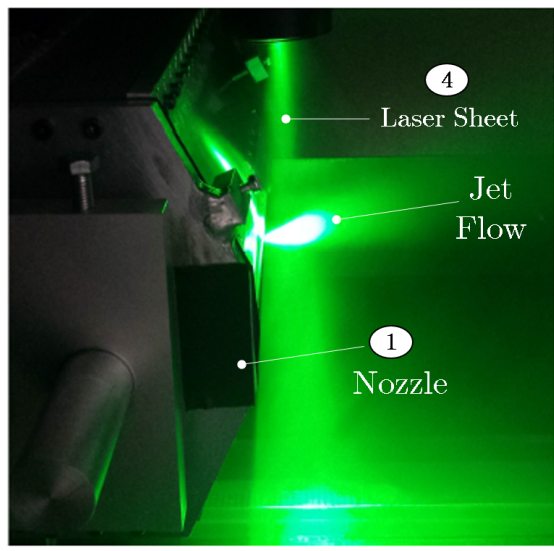

b)

Fig. 5. a) Schematic of the VKI facility used to study the Coanda co-flow fluidic vectoring scheme, with the main components for the flow visualization. b): picture of the set-up during a jet vectoring experiment, showing the nozzle (1), the laser sheet (3) and the deflected jet flow.

pressure, while the velocity field is set to the OpenFOAM pressureInletOutletVelocity condition. This boundary condition allows for correctly modeling both the flow outlet and the entrainment flows by automatically imposing a zero gradient in regions where fluid flows out of the domain and an inlet value (set to $10^{-3}$ ) in the regions where fluid flows enter the domain. The inlet condition for the two injections is a uniform velocity, with the fixed value calculated from the pressure measurement in the experiments. The flow in the channel develops for about $6 h_{p}$ before the release in open pressure, similarly to what happens in the real nozzle. The turbulent kinetic energy is calculated as $k=1.5\left(U_{r} T . I .\right)^{2}$ where the reference velocity $U_{r}$ is taken from the inlet value, and T.I. is the turbulence intensity, set to T.I. $=10 \%$ in agreement with the maximum value obtained from a preliminary experimental characterization of the nozzle via hot wire anemometry. The turbulence dissipation is computed as $\varepsilon=C_{\mu} k^{3 / 2} / l$, with $C_{\mu}=0.09$ and the mixing length $l$ is set to 0.007 times the nozzle exit dimension as suggested in [35].

Concerning the numerical schemes, both the advection and the diffusion terms are discretized with a second order Gauss linear schemes (upwind for the advection terms; corrected for the diffusion terms). The time discretization is the implicit Euler scheme, running with a time-step of about $2 \times 10^{-7} s$ to ensure a CFL number of approximately 0.8 . The physical simulation time to overcome the transitory and reach a steady state solution is generally of the order $0.02 \mathrm{sec}$.

\section{Experimental Methods}

\subsection{Experimental set-up}

The experimental set-up for fluidic control testing developed at the von Karman Institute is shown in the schematic of Figure 5a. The nozzle (1) is composed of two independent chambers, equipped with turbulence grids (2) to uniform turbulence intensity and the span-wise air distribution. These chambers are supplied by two independent air pressure lines, controlled by upstream pressure regulators. A pressure tap is flash mounted on the back of each chamber and connected to two piezoresistive pressure transducers AMS 5812 to acquire the stagnation pressure $P_{p, s}$ of the two injections. Given the chamber discharge coefficient $D_{s, p}$, available from a pressure-velocity calibration via hot-wire anemometry, the velocity of the two injections is computed as $u_{p, s}=D_{s, p} \sqrt{2 P_{p, s} / \rho}$. These pressure transducers have a full scale of about $0-2000 P a$, corresponding to a maximum of $58 \mathrm{~m} / \mathrm{s}$, and an absolute uncertainty of the order of $10 \mathrm{~Pa}$.

A Lasking Nozzle PIVTEC45-M (3), operating with mineral oil (Ondina Shell 917), is used to produce seeding particles in the primary jet and thus to allow for highspeed flow visualization. At the scope, the middle plane of the nozzle is illuminated by a laser sheet (4) of approximately $2 \mathrm{~mm}$ thickness, produced by a light sheet probe (5) LSO, from ILA. The light source is a dual diode-pumped Nd:YLF laser system (Quantronix Darwin Duo 527) running in continuous mode. The images are acquired by a CMOS Photron FASTCAM SA1 (6), placed at about 16 $\mathrm{cm}$ from the test section and equipped with a Tamron CZ$73570-300 \mathrm{~mm}$ zoom objective, leading to a scaling factor of about 21 pixels $/ \mathrm{mm}$. The videos have a maximum size of $5.5 \mathrm{~GB}$, consisting of $n_{t}=12473$ images with a resolution of $n_{p}=512 \times 896$ pixels $^{2}$, sampled at a frequency of $f_{s}=1 \mathrm{kHz}$, corresponding to a acquisition time of about $t \approx 12 s$. The exposure time is set to the maximum possible $\left(\approx 1 / f_{s}\right)$. This, together with the continuous light source and the high seeding concentration, allows for displaying the jet flow as a uniform and bright region, clearly distinguishable from the background. A picture of the facility during an experiment is shown in Fig.5b.

The nozzle facility allows for varying the Coanda radius between $R=2 \mathrm{~mm}$ and $R=5 \mathrm{~mm}$, while the secondary opening can be changed between $h_{s}=0.5 \mathrm{~mm}$ and $h_{s}=1 \mathrm{~mm}$. The primary opening is kept to $h_{p}=1.5 \mathrm{~mm}$ with a gap of $L=1 \mathrm{~mm}$ between the two channels. 


\subsection{Data processing}

As the jet flow appears rather stable in the high-speed flow visualization, each video sequence is down-sampled by a factor of 100 and the centerline of the jet flow is reconstructed from each of the resulting $n_{t}=124$ images.

The centerline detection is carried out with the image processing algorithm proposed in [36,37]. This algorithm consists of three steps. First, each image is horizontally re-contrasted to uniform the light intensity within the jet flow and low-pass filtered with a Gaussian kernel to remove high-frequency noise. An exemplary result of this step is shown in Figure 6a. Second, the grayscale profile in each column is further smoothed via a Savizty-Sgolay filter and the peak of each profile detected. Finally, the jet centerline is constructed as the loci of the grayscale peak in each column and the result from the down-sampled images is time averaged. Three exemplary profiles corresponding to the vertical lines denoted with $x_{1}, x_{2}, x_{3}$ in Figure $6 \mathrm{a}$ are shown in Figure $6 \mathrm{~b}$ as a function of the vertical coordinate and together with the peak detection, marked in black.

The time averaged result is interpolated with a linear fit, from which the thrust vectoring angle is computed. A systematic uncertainty in the fitting process is estimated to be of the order of $\pm 1^{\circ}$. A random relative uncertainty of approximately $\pm 2 \%$, linked to the averaging process, is computed from the standard deviation of the vectoring angle retreived in each of the down-sampled images.
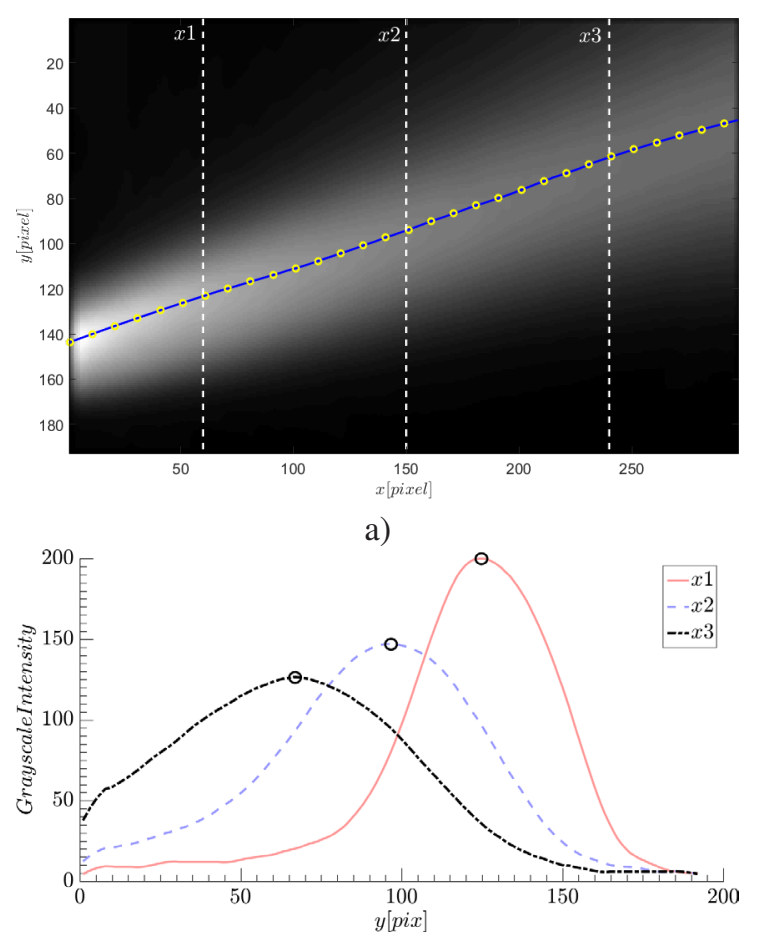

b)

Fig. 6. a) Exemplary image horizontal re-contrasted to uniform the average gray scale value in each column. In each column, the location of the jet centerline is identified as the peak of gray scale intensity. b) Gray scale profiles at three sample locations $x 1=60, x 2=150$ and $x 3=240$ pixels are chosen from the image in a).

\section{Results and Discussion}

\subsection{Numerical Results}

The contour plot of the velocity magnitude and several representative velocity profiles along the radial coordinate are shown in Figure.5.1 for four numerical simulations. These test cases include a reference test with $u_{s}=57 \mathrm{~m} / \mathrm{s}$ and $u_{p}=0 \mathrm{~m} / \mathrm{s}$ (5.1a) and three active control cases with blowing ratios of $\beta_{M}=1.47,0.48,0.12$ (Figure $5.1 \mathrm{~b}, \mathrm{c}, \mathrm{d}$ ).

The geometrical parameters are $h_{s}=0.5 \mathrm{~mm}, h_{p}=$ $1.5 \mathrm{~mm}$ and $R=5 \mathrm{~mm}$, with $L=1 \mathrm{~mm}$, while the velocity of the primary jet is kept at $u_{p}=38 \mathrm{~m} / \mathrm{s}$. The velocity profiles extracted along $\theta=10^{\circ}, 30^{\circ}, 50^{\circ}$ and $70^{\circ}$ and scaled with respect to the average velocity in eq.(11), with $\xi=\left(h_{p} / h_{s}\right) \beta_{M}$ as described in Section 2.4. The radial coordinate ranges from $r=R$ (at the Coanda wall) to the half width of the primary jet, namely $r=R+h_{s}+L+h_{p} / 2$ and it is shifted and scaled to the range $\hat{r} \in[0,1]$.

The evolution of the pressure gradient at the wall $P(\theta)=\partial_{r} p(\theta) / p(0)$ and the average velocity $G(\theta)=$ $u_{A}(\theta) / u_{A}(0)$ is shown in Figure $8 \mathrm{a}$ and $8 \mathrm{~b}$ respectively. Here, the average velocity is taken as the integral average in the radial coordinates, that is

$$
u_{A}(\theta)=\int_{\hat{r}} u(\hat{r}, \theta) d \hat{r}
$$

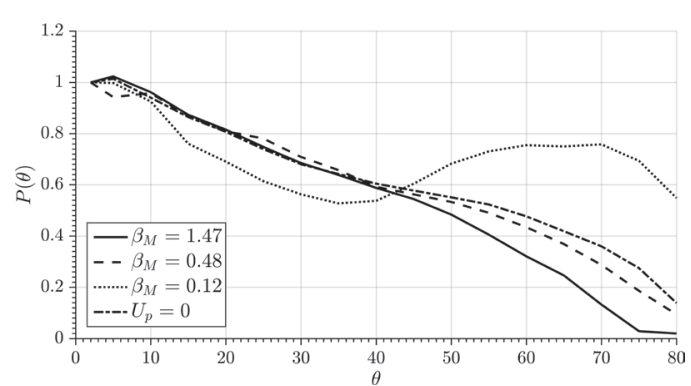

a)

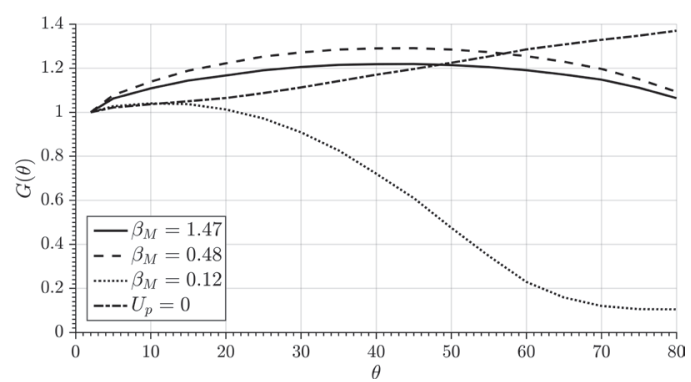

b)

Fig. 8. Evolution of the radial pressure gradient $P(\theta)$ along the Coanda surface (a) and the averaged velocity $G(\theta)$ as function of the angular coordinate $\theta$ for the four numerical test cases shown in Figure 5.1.

Concerning the reference test case in Figure 5.1a with no primary injection $\left(u_{p}=0 \mathrm{~m} / \mathrm{s}\right)$, the velocity profiles display the classical shape of a wall jet, that is a combination of a boundary layer profile in the bottom part, close to the wall, and a free jet profile in the upper part. 
a)

$$
\begin{gathered}
u_{p}=0 \\
u_{s}=57 \mathrm{~m} / \mathrm{s} \\
\beta_{M}=[-] \\
\xi=1 \\
u_{M}=57 \mathrm{~m} / \mathrm{s}
\end{gathered}
$$

b)

$$
\begin{gathered}
u_{p}=38 \mathrm{~m} / \mathrm{s} \\
u_{s}=57 \mathrm{~m} / \mathrm{s} \\
\beta_{M}=1.47 \\
\xi=1 \\
u_{M}=57 \mathrm{~m} / \mathrm{s}
\end{gathered}
$$
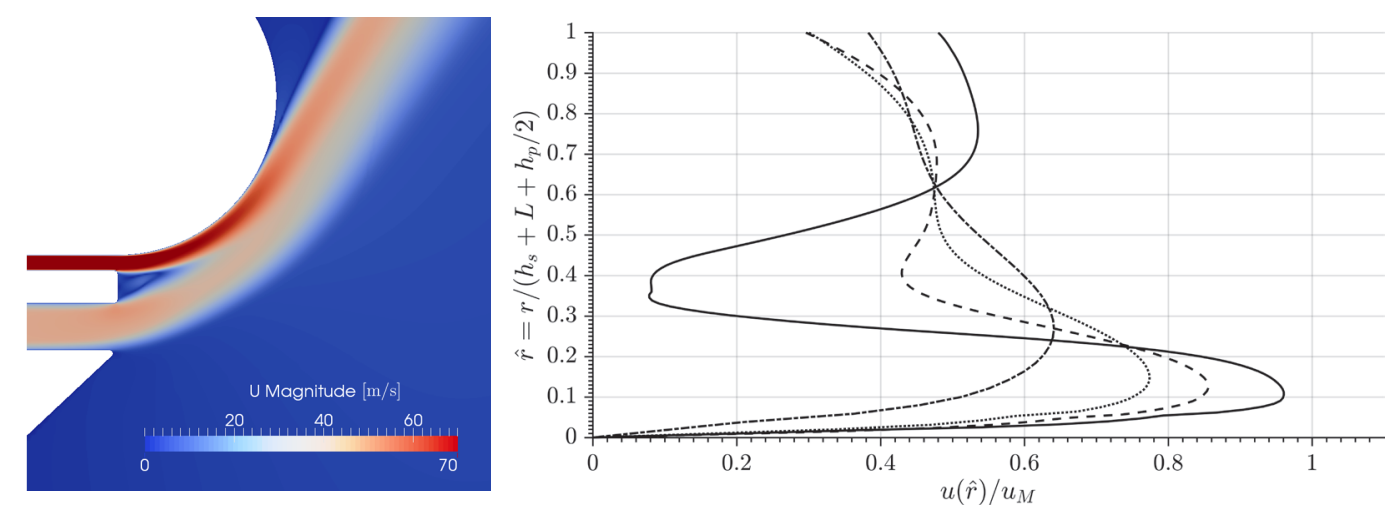

c)

$u_{p}=38 \mathrm{~m} / \mathrm{s}$ $u_{s}=45.6 \mathrm{~m} / \mathrm{s}$

$\beta_{M}=0.48$

$\xi=1$

$u_{M}=45.6 \mathrm{~m} / \mathrm{s}$
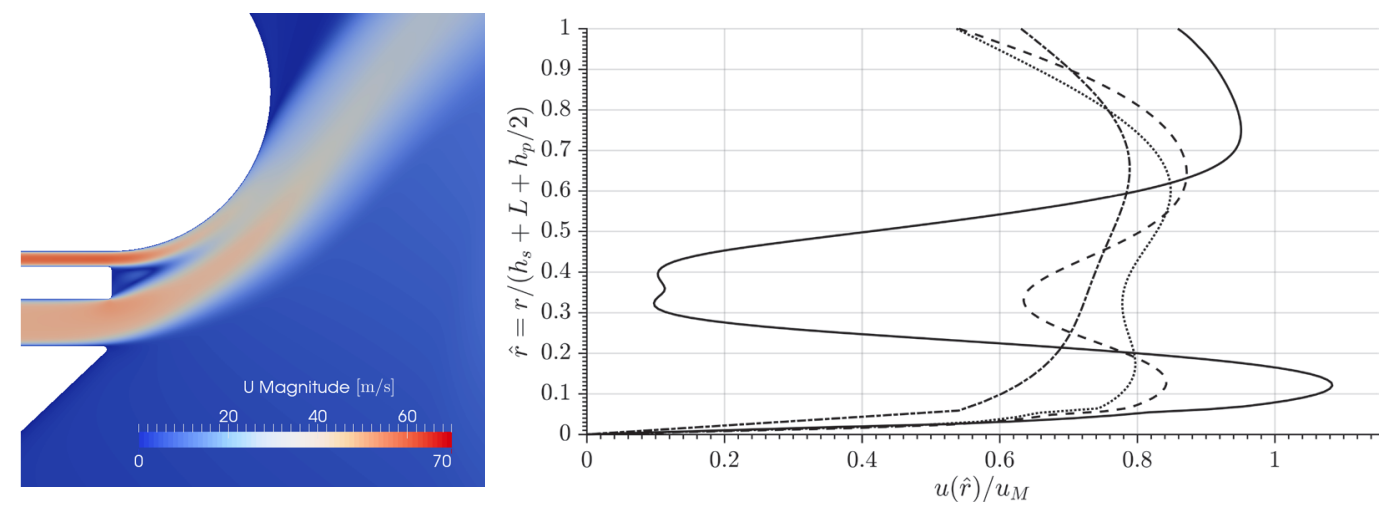

d)

$u_{p}=38 \mathrm{~m} / \mathrm{s}$

$u_{s}=22.8 \mathrm{~m} / \mathrm{s}$

$\beta_{M}=0.12$

$\xi=0.36$

$u_{M}=33 \mathrm{~m} / \mathrm{s}$
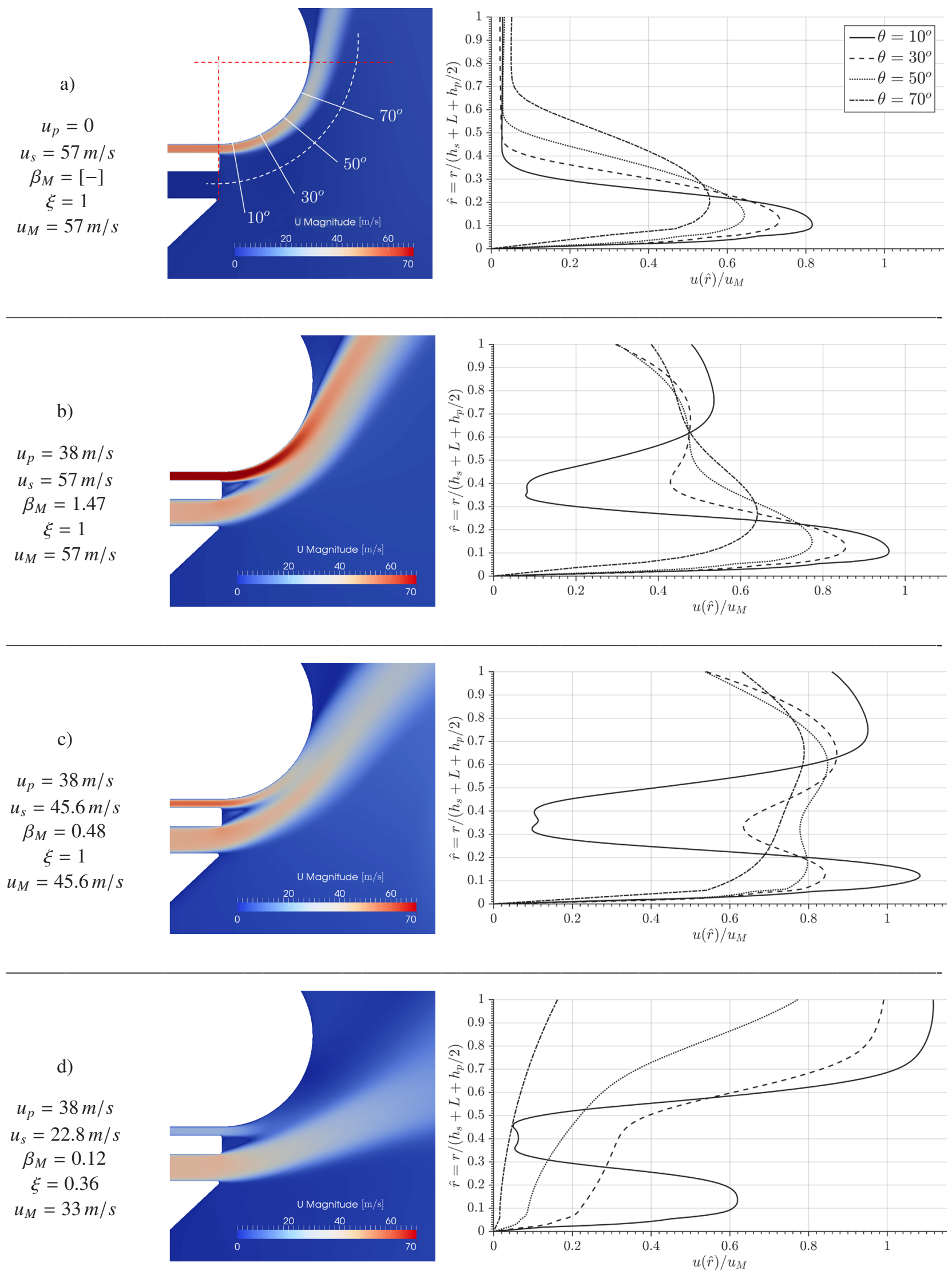

Fig. 7. Contour of the velocity magnitude at the nozzle outlet (left) and evolution of the velocity profiles (right) for four numerical test cases. The geometrical parameters are $h_{s}=0.5 \mathrm{~mm}, h_{p}=1.5 \mathrm{~mm}, R=5 \mathrm{~mm}$ and $L=1 \mathrm{~mm}$; the operating conditions are indicated on the left, including the average velocity $u_{M}$ from eq.(11) that is used to scale the velocity profiles on the right. 
The effects of turbulent and viscous diffusion is that of decreasing the maximum velocity and to increase the width of the jet. As a result, the pressure gradient at the wall (dash-dotted line in Figure 8a) drops almost linearly with the angular coordinate, while the average velocity (dash-dotted line in Figure 8b) increases. That is also visible from the velocity profiles: the decay of the maximum velocity is largely compensated by the thickening of the jet flow due to the entrainment of surrounding fluid.

When both injections are active, the trend of these macroscopic quantities remains rather unchanged for a wide range of momentum ratios. Figure $5.1 \mathrm{~b}$ reproduces the same results of Figure5.1a for a momentum ratio as large as $\beta_{M}=1.47$, that is well beyond the saturation condition. This implies $\xi=1$ and thus $u_{M}=u_{s}$ from equation (11). The velocity peak is only slightly influenced by the momentum ratio, despite the considerable broadening of the velocity profiles due to the momentum exchange. The pressure gradient (continuous line in Figure 8a) at the wall remains close to the reference case, departing slightly only for $\theta>40^{\circ}$. The evolution of the average velocity (continuous line in Figure $8 b$ ) displays a slight parabolic shape, but remains of the order of $G(x) \approx 1$. Decreasing the momentum ratio to $\beta_{M}=0.48$ does not significantly alter these behavior, as visible from Figure 5.1c and from the dashed line in Figures 8a,b.

These results prove that the model coefficient in eq.(14) remains independent from the momentum ratio $\beta_{M}$ over a large range of operating parameters.

Such simplification, however, reveals its limit at very low momentum ratio, as can be seen from Fig.5.1c and the dotted line in Figure8a-b, regarding the numerical test case with $\beta_{M}=0.12$. In this case, the evolution of the average velocity is mostly governed by the velocity of primary jet flow, as correctly predicted by eq (12). However, the separation of the secondary jet occurs much sooner than in the previous cases and this reduces the range $x \in\left[0, x^{*}\right]$ over which the pressure gradient driving the vectoring is acting. The limits of the integral in eq.(14), therefore the model coefficient (14), depend on the momentum ratio at the limit $\beta_{M} \ll \beta_{M}^{S}$. At larger momentum ratios, on the other hand the simplification of a constant model coefficient in eq.(14) appears largely justified.

\subsection{Experimental Validation}

Three geometrical configurations have been analyzed over a wide range of momentum ratios. The first two investigate the impact of the secondary opening $h_{s}$, changing from $h_{s}=0.5 \mathrm{~mm}$ to $h_{s}=1 \mathrm{~mm}$ while keeping the primary opening at $h_{p}=1.5 \mathrm{~mm}$ and the Coanda radius to $R=5 \mathrm{~mm}$. The third case investigates the impact of the Coanda radius, reduced to $R=2 \mathrm{~mm}$, while keeping $h_{s}=0.5 \mathrm{~mm}$ and $h_{p}=1 \mathrm{~mm}$. All the cases are performed with two velocities in the primary jet flow, namely $u_{p}=28 \mathrm{~m} / \mathrm{s}$ and $u_{p}=38 \mathrm{~m} / \mathrm{s}$. Four exemplary flow visualizations for the cases with $u_{p}=28 \mathrm{~m} / \mathrm{s}$ at different momentum ratios are shown in Figure 9, together with the automatic centerline detection.
The measured vectoring coefficient as a function of the momentum ratio is shown in Figures 10a,b,c, and compared to the prediction of the empirical model in eq.(16) taking the model coefficient in eq.(14) as $C=0.39\left(h_{p} / h_{s}\right)$. For the three investigated geometrical configurations, the model prediction are in close agreement with the experimental measurements. In particular, a comparison between Figure 10a and Figure 10b reveals the impact of the ratio of the two openings $h_{s} / h_{p}$, which, with remarkable agreement with the model prediction, sets the momentum ratio at the saturation at $\beta_{M}^{S} \approx h_{s} / h_{p}$. A comparison between Figure 10-b with Figure 10-c, on the other hand, shows the limited influence of the Coanda radius, in agreement with the formulation of the empirical model.

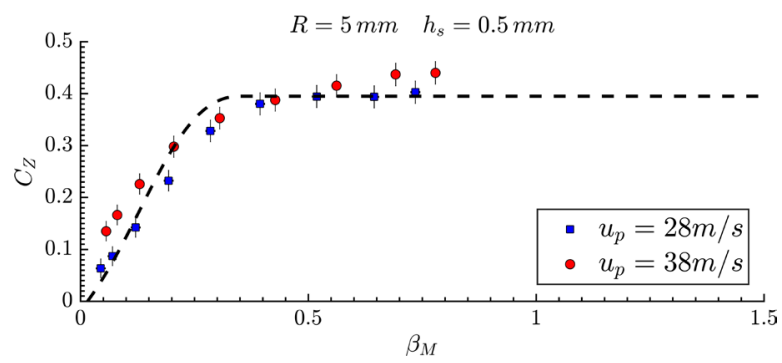

a)

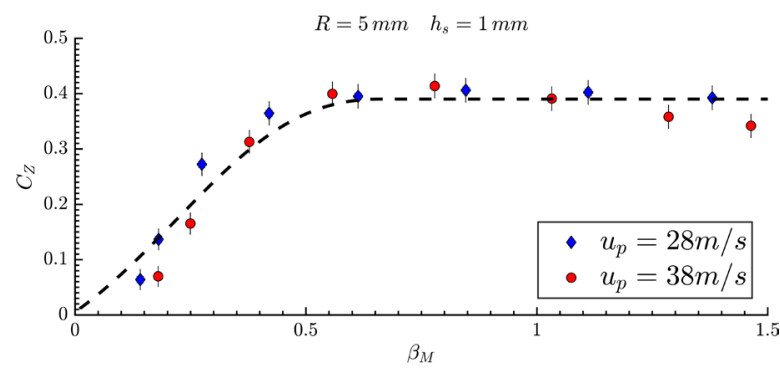

b)

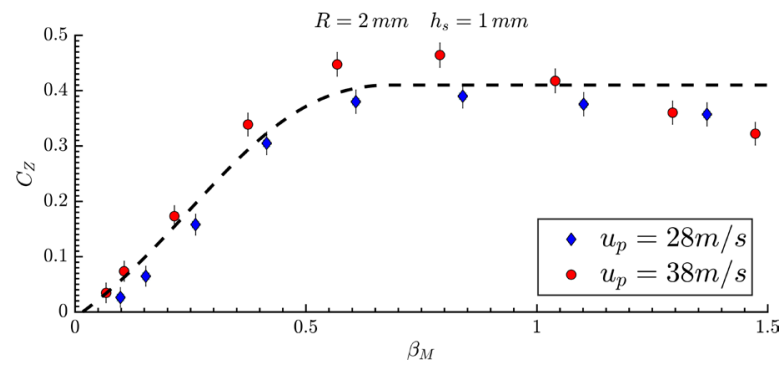

c)

Fig. 10. Thrust vectoring coefficient $C_{Z}$ as a function of the momentum ratio $\beta_{M}$ for three geometrical configurations, comparing the prediction of the proposed empirical model with two sets of experimental points with $u_{p}=28 \mathrm{~m} / \mathrm{s}$ and $u_{p}=38 \mathrm{~m} / \mathrm{s}$. The primary opening is fixed at $h_{p}=1.5 \mathrm{~mm}$, the Coanda radius and the secondary opening are indicated in the plot title.

Finally, the influence of the primary velocity appears reasonably negligible for the large Coanda radius (10a,b), once again in agreement with the empirical model, but not at the lowest radius (10c). In this case a proper saturation is not reached at the highest velocity, but the vectoring performances appear to decrease after having reached 

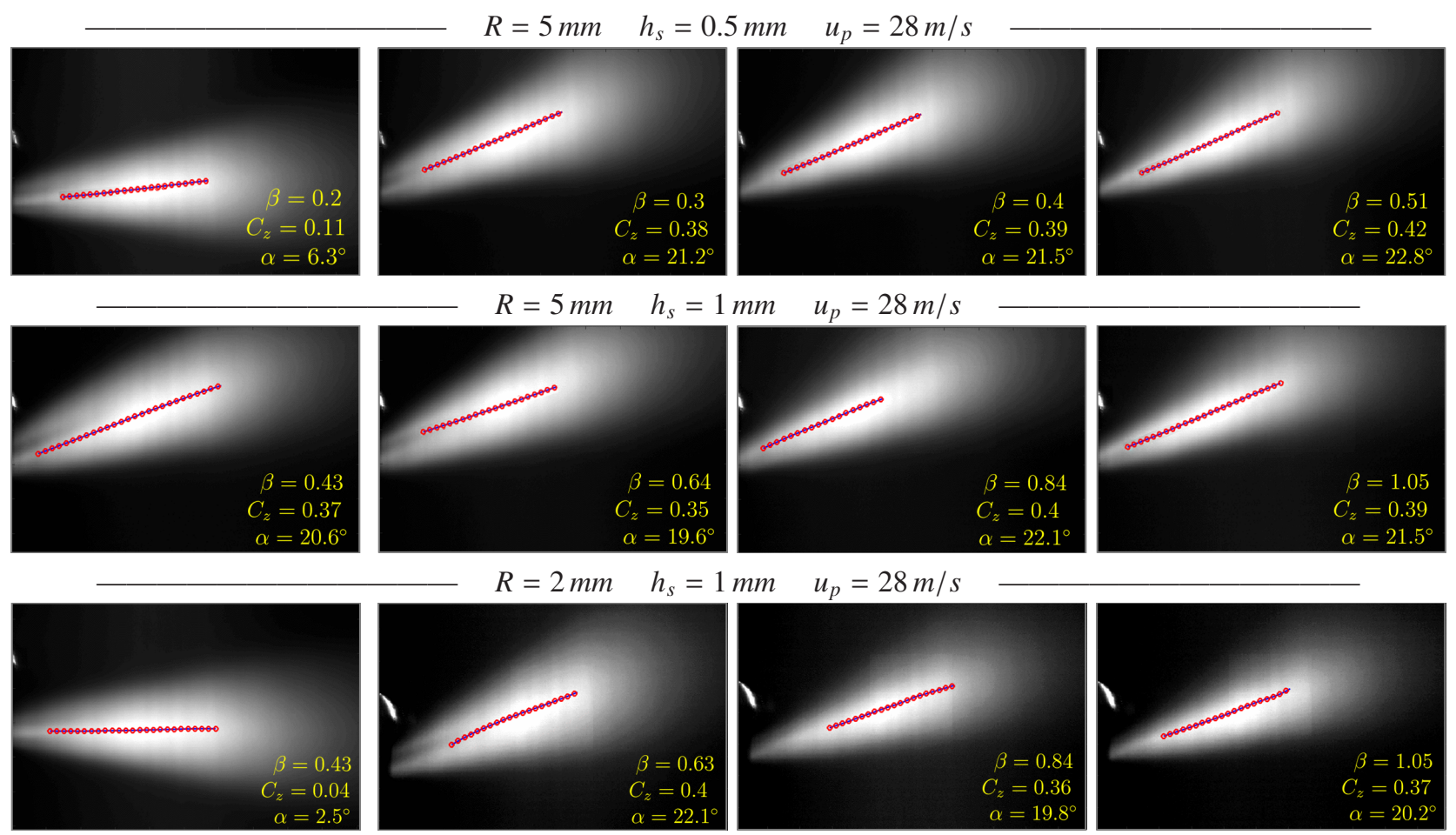

Fig. 9. Set of four down-sampled images and jet centerline detection for the three geometrical configurations investigated experimentally and for the primary jet flow at $u_{p}=28 \mathrm{~m} / \mathrm{s}$. The measured vectoring angle and coefficient are indicated in each picture, together with the mass ratio $\beta$ in equation.(1).

the maximum value. Such behavior, not predictable by the proposed model, could be due to the separation of the secondary jet flow, which might become more strongly linked to the momentum ratio for higher curvature parameters $h_{s} / R$. In this case, the assumption of constant $C$ in eq.(14)- therefore the assumption of linear dependency of the transversal pressure gradient versus the momentum ratio- is clearly too restrictive.

\section{Conclusions}

An experimental, numerical and theoretical investigation of a co-flow Coanda fluidic design for the vectoring of a slot, incompressible jet has been presented. This design consists of a secondary injection, parallel to the primary, and a cylindrical surface to exploit the Coanda effect. An empirical model for predicting the vectoring performance as a function of the geometrical and the operating parameters has been formulated. This model describes the vectoring process as the result of a transversal pressure gradient, governed by the Coanda effect, and inertia, governed by the momentum exchange between the two jet flows.

The simplifying hypothesis postulated in the model derivation are validated numerically by means of $2 \mathrm{D}$ URANS simulations and the model predictions are compared to a large set of experimental data from flow visualization and image processing.

Within the investigated range of geometrical parameters, the results have shown that the jet vectoring is mostly governed by the ratio of the secondary to the primary opening of the jet flows, rather than the Coanda radius.
This parameter defines in fact the momentum ratio above which the achievable vectoring angle saturates. For three different geometrical configuration and two primary velocities, the prediction of the model are found to be in close agreement with the experimental data over a large range of momentum ratios.

\section{Acknowledgments}

The authors gratefully acknowledge the Belgian FNRS for supporting M. A. Mendez with a FRIA grant and ArcelorMittal, for the financial support and for the permission to publish the material presented. We finally thank D. Resende and L. Paoli for their contribution in a preliminary experimental and the numerical work, conducted in the framework of a Short Training Program (STP) at the von Karman Institute for Fluid Dynamics.

\section{List Of Symbols}

\section{Notation and Subscripts}

$\partial_{y}(\bullet) \quad$ partial derivative along $y$

$\partial_{r}(\bullet)$ partial derivative along $r$

$\partial_{t}(\bullet) \quad$ partial derivative in time

$p \quad$ primary injection

$s \quad$ secondary injection 


\section{Symbols}

$C_{z} \quad$ thrust vectoring coefficient

$D$ nozzle discharge coefficient

$f_{s} \quad$ image acquisition frequency

$G \quad$ averaged velocity decay

$h$ nozzle aperture

$L$ distance between the two jet apertures

$n_{p} \quad$ image resolution

$n_{t} \quad$ number of images

$$
\text { pressure }
$$

wall pressure gradient decay

radial coordinate

Coanda radius

jet velocity

averaged profile velocity along $r$

averaged velocity from eq.(12)

horizontal coordinate

jet potential core length

vertical coordinate

vectoring angle

mass flux ratio

momentum ratio

critical $\beta$

saturation $\beta$

curvilinear coordinate

angular coordinate

mixing coefficient

air density
9. Smith, B.L., Glezer, A., Bull. Am. Phys. Soc. 39, 1894 (1994)

10. Smith, B.L., Glezer, A., AIA Journal 43, 2117 (2005)

11. Heo, J.-Y., Yoo, K.-H., Lee, Y., Sung, H.-G., Cho, S.H., Jeon, Y.-J., Journal of Propulsion and Power 28, 858 (2012)

12. Strykowski, P. J., Krothapalli, A., Forliti, D. J., AIA Journal 34, 2306 (1996)

13. Flamm, J. D., Experimental study of a nozzle using fluidic counterflow for thrust vectoring, in 34th Joint Propulsion Conferences (1998), Vol. 98-3255

14. Hunter, C.A., Deere, K.A., Computational Investigation of Fluidic Counterflow Thrust Vectoring, in 35th Joint Propulsion Conference and Exhibit, Joint Propulsion Conferences (1999), Vol. 99-2669

15. Humes, Z., Smith, B.L., Minichiello, A.L., Particle sosorting by aerodyamic vectoring, in Bull. Am. Phys. Soc. (2005)

16. B. Newman, Boundary Layer and Flow Control Principles and Applications (Pergamon Press Inc., 1961), Vol. 1, chap. The deflection of Plane Jets by Adjacent Boundaries- Coanda Effect, pp. 232-264

17. Bradshaw, P., Tech. rep., AGARDograph n 169 (1973)

18. Carpenter, P.W., Green, P.N., Journal of Sound and Vibration 208, 777 (1997)

19. Smith, B.L., Glezer, A., J. Fluid Mech 458, 1 (2002)

20. Bremhorst, K., Hollis, P.G., AIAA Journal AIAA $97-$ 3348, 2043 (1990)

21. Pack, L.G., Seifer, A., Journal of Aircraft 38, 486 (2001)

22. Al-Asady, A.A.A., Abdullah,A.M., NJES 20, 5 (2017)

23. Saghafi, F., Banazadeh, A., The Aeronautical Journal 3117, 17 (2008)

24. Saghafi, F., Banazadeh, A., Coanda Surface Geometry Optimization for Multi-Directional Co-flow Fluidic Thrust Vectoring, in Proceedings of ASME Turbo Expo 2009 (2009)

25. Song, M.J., Yoon, S.H., Chang, H.B., Cho, Y.H., Lee, Y, A Study on the Performance of the Fluidic Thrust Vector Control Utilizing Supersonic Coanda Effects, in 5th Symposium on Integrating CFD and Experiments in Aerodynamics (2012), pp. 253-261

26. Zmijanovic, V., Lago, V., Leger, L, Depussay, E., Sellam, M., Chpoun,A., Progress in Propulsion Physics 4, 227 (2013)

27. Bharathwaj, R, Giridharan, P, Karthick,K., Prasath, C.H., Prakash, K.,M., IOP Conf. Ser.: Mater. Sci. Eng. 149, 012210 (2016)

28. Trancossi, M., Dumas, A., SAE Technical Papers (2011)

29. Kirshner, J.M., Katz, S., Design Theory of FlFluidic Components (Academic Press, 1975)

30. Dumitrache, A., Frunzulica,F., Ionescu,T.C. , in Nonlinearity, Bifurcation and Chaos - Theory and Applications, edited by Awrejcewicz, J., Hagedorn, P. (InTech, 2012)

31. Reichardt, H., Z. Angew. Math. Mech. 21 (1941) 
32. Schlichting, H., Boundary-Layer Theory (McGrawHill, 1960)

33. S.L. Abramovich, G.N., The Theory of Turbulent Jets (The MIT Press, 1963)

34. Menter, F.R., AIAA Journal 32, 1598 (1994)

35. Versteeg, H.K., Malalasekera, W., An Introduction to Computational Fluid Dynamics (Longman Scientific \& Technical, 1995)
36. Mendez, M.A., Buchlin, J.-M., Quantitative flow visualization of confinement-driven instabilities of an impinging slot jet, in 11th International Symposium on Particle Image Velocimetry (PIV2015) (Santa Barbara, California, US, 2015)

37. Mendez, M.A., Scelzo, M.T., Buchlin, J.-M., Exp Therm Fluid Sci 91, 256 (2018) 